\title{
The hamiltonian study of supersymmetric Yang-Mills quantum mechanics
}

\author{
M. Trzetrzelewski ${ }^{\mathrm{a} *}$ \\ ${ }^{a}$ M. Smoluchowski Institute of Physics, Jagiellonian University, \\ Reymonta 4, 30-059 Cracow, Poland
}

The hamiltonian formulation of Supersymmetric Yang-Mills quantum mechanics (SYMQM) is discussed. We focus on the Fock space formulation of the models since it is convenient for the numerical analysis, however some novel analytical results are also pointed out.

\section{A brief history of SYMQM}

Supersymmetric Yang-Mills quantum mechanics by definition are $\mathcal{N}=1$ supersymmetric YangMills field theories reduced from $D=d+1$ spacetime dimensions to $0+1$ dimensions.

Almost thirty years ago the purely bosonic part of SYMQM was conceived by Bjorken [1] in the zero volume limit of YM theories. The subject was then pushed forward by Lüsher [2] resulting in small volume expansion of glueball masses for the $S U(2)$ gauge group. At the same time the model was considered by Hoppe in his Phd. thesis as the regularized description of relativistic membrane [3]. The supersymmetric formulation of YMQM was first discussed by Cloudson and Halpern [4]. Later on the supermembrane was formulated [5]6] as well as its regularized description [7] resulting precisely in SYMQM. The interest in quantum formulation of (super)membranes was originally motivated by particle physics i.e. membranes were believed to describe elementary particles, the idea first put forward by Dirac 8 . However, it turns out that the spectrum of the hamiltonian of SYMQM is continuous hence the supermembrane is unstable 9. Almost a decade later this set back was interpreted as good news since this time SYMQM were conjectured [10] to describe M-theory. The continuous spectrum corresponds now to the scattering states on M-theory side and there is no contradiction since M-theory is a second quantized theory.

\footnotetext{
*I thank the organizers of the Cargse Summer School, May
} 22-June 3, 2006 for their kind invitation and support.

\section{The Fock space approach}

Supersymmetry imposes some constraints on dimensionality of SYM theories ( hence SYMQM ) namely $D=2,3,4,6,10$. SYMQM consists of real bosonic variables $x_{a}^{i}$ and complex fermionic variables $\psi_{a}^{\alpha}$ ( for $D=10$ the 16 Majorana-Weyl fermions can be composed into the 8 complex ones ). Here $a=1, \ldots, N^{2}-1$ is a color index i.e $x_{a}^{i}$ and $\psi_{a}^{\alpha}$ are in the adjoint representation of $S U(N)$ while $i=1, \ldots, D-1$ and $\alpha=1, \ldots, D-2$ are spatial and spinor indices respectively. It seems that there is a mismatch between bosonic and fermionic degrees of freedom however, while performing the dimensional reduction the Gauss law becomes a global $S U(N)$ invariance. In the subspace of $S U(N)$ singlets the degrees of freedom match. The hamiltonian of SYMQM is then [4]

$H=\frac{1}{2} \pi_{a}^{i} \pi_{a}^{i}+\frac{1}{4} g^{2}\left(f_{a b c} x_{b}^{i} x_{c}^{j}\right)^{2}+H_{F}$,

where $f_{a b c}$ are $S U(N)$ structure constants, $\pi_{a}^{i}$ are conjugate to $x_{a}^{i},\left[x_{a}^{i}, \pi_{b}^{j}\right]=i \delta_{a b} \delta^{i j}$ and $H_{F}$ is the fermionic part which schematically is $H_{F}=$ igx $\bar{\psi} \Gamma \psi$, where $\Gamma$ are Dirac gamma matrices in corresponding dimension.

We now introduce, in the standard fashion, the bosonic creation and annihilation operators $a_{a}^{\dagger}{ }_{a}^{i}$, $a_{a}^{i}$ and analogously fermionic operators ${f^{\dagger}}_{a}^{\alpha}, f_{a}^{\alpha}$ although in this last case the construction depends on the dimensionality ( see [12]13] for conventions ). Any state $|s\rangle$ in Fock space is now a linear combination of products of creation opera- 
tors acting on the Fock vacuum $|0\rangle$, so that the resulting state $|s\rangle$ is gauge invariant. For numerical analysis we now introduce a cutoff $n_{B_{\max }}$ and truncate the Hilbert space so that the states have no more then $n_{B_{\max }}$ number of bosonic quanta. Then we compute the matrix elements of (1) and diagonalize the truncated matrix $H^{\left(n_{B_{\max }}\right)}$. The resulting eigenvalues converge very fast to the exact eigenvalues of the hamiltonian provided the spectrum is discrete. In the case of continuous spectrum the situation is more subtle. We refer to [11] where it is discussed in details.

The method just described was applied to $D=$ 4, $S U(2)$ SYMQM resulting in very precise evaluation of the spectrum and the Witten index [12]. The analogous calculation for $D=10$ SYMQM is difficult to perform since in this case the number of states from Fock space grows extremely fast with $n_{B_{\max }}$ [13]. The remedy at this point could be the additional $S O(9)$ invariance. It is then possible that the numerical analysis of these models is within reach once we work in sectors with given $S O(9)$ angular momentum.

\section{Exact results and large $\mathbf{N}$}

While for SYMQM with $D>2$ there are no exact solutions the $D=2$ case is different. The system is much simpler then the higher dimensional relatives since it is where the quartic potential term in the hamiltonian, vanishes. The hamiltonian is simply $H=\frac{1}{2} \pi_{a} \pi_{a}$ however, the model is not free due to the Gauss law. The exact solutions in the bosonic sector are known for $S U(2)$ 4 and arbitrary $S U(N)$ [14 groups. The disadvantage of these solutions is the absence of explicit $N$ dependence hence the $N \rightarrow \infty$ limit is difficult. Moreover, the existing solutions are not general ones except for the $D=2, S U(2)$ SYMQM. It is possible to overcome these difficulties. In 15 we have found a class of solutions which differ from the existing ones. Moreover, their large $N$ limit is possible although it turns out that one has to be very careful in performing the limit due to the distinguished role of the bilinear operators $x_{a} x_{a}$. The result reads

$$
|p\rangle=e^{-p^{2} r^{2} / 2 N^{2}}|v\rangle, \quad r=\sqrt{x_{a} x_{a}}, \quad N>>1,(2)
$$

where $|v\rangle$ is the vacuum state and $p$ is the momentum. We see that all the $1 / N^{k}$ terms are present in the Taylor expansion of the large $N$ solutions. It can be argued that they are all of equal importance hence the "ordinary" large $N$ techniques may fail when the hamiltonian considered has continuous spectrum. However, this is precisely the case for all SYMQM. Moreover, if we put $g=0$ in (1) we obtain the class of large $N$ solutions, having the form of products of (2), for SYMQM in arbitrary dimensions.

\section{Conclusions}

The numerical approach to SYMQM presented here is very encouraging. Although so far applied only to $D=4$ case we believe that it will finally give us the nonperturbative results also in the $D=10$ system.

A careful large $N$ analysis indicates that the $1 / N^{k}$ terms may play an important role which is in contrast with the majority of large $N$ computations.

\section{Acknowledgments}

This work was supported by the the grant of Polish Ministry of Science and Education no. P03B 02427 ( 2004 - 2007 ) and N202 044 31/2444 ( 2006-2007) and the Jagiellonian University Estreicher foundation.

\section{REFERENCES}

1. J.D. Bjorken, Elements of quantum chromodynamics, SLAC-PUB-2372, 1979. 139pp.

2. M. Lüscher, Nucl. Phys. B219, (1983), 233;

3. J. Hoppe, PhD Thesis MIT, (1982), unpublished;

4. M. Claudson and M. B. Halpern, Nucl. Phys. 250 (1985), 689, 6189;

5. J. Hughes, J. Liu, J. Plochinski, Phys. Lett, B180, (1986), 370;

6. E. Bergshoeff, E. Sezgin, P. K. Townsend, Phys. Lett. B189, (1987), 75;

7. D. de Wit, J. Hoppe, H. Nicilai, Nucl. Phys. B305[FS23], (1988), 545-581;

8. P. A. M. Dirac, Proc. Roy. Soc. A268, (1962), 57 ; 
9. B. de Wit, M. Lüscher and H. Nicolai, Nucl. Phys. B320, (1989), 135;

10. T. Banks, W. Fischler, S. Shenker and L. Susskind, Phys. Rev. D55, (1997), 6189;

11. M. Trzetrzelewski, J. Wosiek, Acta Phys. Polon. B35 , (2004), 1615-1624;

12. M. Campostrini, J. Wosiek, Nucl.Phys. B703, (2004), 454-498;

13. J. Wosiek, Phys. Lett. B619, (2005), 171-176;

14. S. Samuel, Phys. Lett B411, (1997), 268;

15. M. Trzetrzelewski, J. Math. Phys. 48 , 012302, (2007); 